\title{
Enseigner sa discipline dans une langue 2. Nouvelle formation continue pour le second degré en Alsace
}

\section{Anemone Geiger-Jaillet et Gérald Schlemminger}

\section{OpenEdition}

\section{Journals}

Édition électronique

URL : http://journals.openedition.org/trema/3250

DOI : $10.4000 /$ trema.3250

ISSN : 2107-0997

Éditeur

Faculté d'Éducation de l'université de Montpellier

Édition imprimée

Date de publication : 1 novembre 2014

Pagination : $138-153$

ISSN : 1167-315X

Référence électronique

Anemone Geiger-Jaillet et Gérald Schlemminger, « Enseigner sa discipline dans une langue 2.

Nouvelle formation continue pour le second degré en Alsace ». Tréma [En ligne], 42 I 2014, mis en ligne

le 16 juin 2015, consulté le 01 mai 2019. URL : http://journals.openedition.org/trema/3250 ; DOI :

10.4000/trema.3250

Ce document a été généré automatiquement le 1 mai 2019.

Trema 


\title{
Enseigner sa discipline dans une langue 2. Nouvelle formation continue pour le second degré en Alsace
}

\author{
Anemone Geiger-Jaillet et Gérald Schlemminger
}

\section{Quelques repères}

1 Au niveau de l'enseignement ainsi que de la formation des enseignants, il faut distinguer deux grands dispositifs en France : certaines langues régionales bénéficient d'un dispositif bilingue appelé «français + langue régionale » allant de la maternelle au baccalauréat (voir infra), alors que l'enseignement dit bilingue est davantage réservé aux langues de diffusion internationale et ne commence (sauf exception) qu'à partir de la $4 \mathrm{e}$ année de collège. Font exception à cette règle les sections internationales un peu partout en France.

Historiquement, l'enseignement bilingue français allemand a été introduit par le Traité de l'Élysée (1963). Afin de renforcer la coopération franco-allemande, les deux États décident, entre autres, la création de trois lycées franco-allemands où l'histoire et la géographie - entre autres - sont enseignées dans la langue du partenaire ${ }^{1}$. Le développement concomitant de l'enseignement de disciplines enseignées en L2 dans les langues régionales dès le primaire après 1983 donne une impulsion à un tel type de pédagogie. Ce développement s'effectue dans les régions qui bénéficient d'une reconnaissance d'une langue régionale par le Ministère : il s'agit du basque, du breton, du catalan, du corse, du créole, de l'occitan-langue d'oc et des langues régionales d'Alsace et des pays mosellans ${ }^{2}$. Se rajoutent dans une moindre mesure le gallo et le flamand. C'est dans les années 1990 que ce type d'enseignement connaît son véritable essor dans les « sites bilingues » (il s'agit de classes spécifiques à l'intérieur des écoles monolingues de la République). 
3 Des « sections européennes » ou de « section de langue orientale » ${ }^{3}$ ouvrent en 11 langues différentes en France en 1992 à partir du collège, elles font l'objet d'une indication portée sur le diplôme du baccalauréat général, technologique ou professionnel. La filière ABIBAC, mot composé de l'allemand Abitur et du baccalauréat français, est proposée dans un nombre limité de lycées suite à l'accord du 31 mai 1994 entre l'Allemagne et la France ${ }^{4}$. D'autres baccalauréats à double délivrance suivent: BACHIBAC (baccalauréat français et bachillerato espagnol) en 2008, ESABAC (baccalauréat français et Esame di stato italien) en 2009.

4 Un second critère de classification de ces quatre dispositifs est le volume horaire d'enseignement dans une autre langue que la langue première de la scolarité, consacré à des contenus disciplinaires. Selon ce critère, nous pouvons distinguer trois dispositifs principaux :

- L'enseignement « bilingue » partiel : les sections européennes ou de langues orientales, les classes des trois lycées franco-allemands ;

- L'enseignement bilingue français-langue régionale d'immersion à parité horaire des langues (de type 50-50 en primaire) ;

- L'enseignement bilingue au-delà de la parité dans des classes associatives privées hors contrat avec l'éducation nationale.

Un troisième critère, non historique mais fonctionnel, consiste à distinguer les dispositifs selon les paramètres de la psycholinguistique et de la didactique (Geiger-Jaillet, 2005a) : précocité, continuité, volontariat, parité et instrumentalisation des langues dans l'enseignement. Nous les expliquerons brièvement :

6 Toutes les formes d'enseignement historiquement présentes sur le territoire français appliquent le principe d'instrumentalisation : la langue sert à transmettre des contenus disciplinaires hors de la langue. Mais seuls les enseignements bilingues d'immersion à parité horaire ou d'immersion au-delà de la parité appliquent les principes de la psycholinguistique (précocité du début de l'apprentissage en maternelle, volontariat de la part des élèves et de leurs enseignants...). Dans le second degré, les cursus bilingues atteignent entre 9 et 12 heures hebdomadaires en langue cible et DEL2. Ce n'est certes plus la parité horaire de l'école primaire, mais ces filières constituent la continuité des enseignements bilingues à parité horaire dans la plupart académies concernées par des langues régionales en France. La question posée par la didactique est de savoir si le terme «bilingue » peut être employé pour tous les dispositifs décrits (lycées franco-allemands, sections internationales, sections européennes, classes bilingues de langues régionales et à parité horaire). Mais cette approche dépasse le cadre de cette étude.

7 Lorsqu'il est question aujourd'hui d'un enseignement bilingue, celui-ci se déroule actuellement grosso modo dans les conditions suivantes :

- Les élèves assistant à ces cours ne sont pas (encore) bilingues, mais le deviendront au cours de leur scolarité ;

- En règle générale, l'enseignement ne se fait pas en deux langues, ni simultanément (les deux langues utilisées à l'intérieur d'un même cours), ni alternativement (le même sujet traité dans la première, puis la seconde langue ou l'inverse).

$8 \mathrm{Au}$ lieu de parler d'un enseignement bilingue, qu'il soit d'immersion partielle, d'immersion de type 50-50 ou d'immersion plus forte, il est plus approprié de parler d'une discipline enseignée en langue 2 (DEL2) ${ }^{5}$. Il peut s'agir de toute discipline dispensée en collège ou lycée. Cette dénomination offre l'avantage, en cas de besoin, de pouvoir situer 
la discipline dans l'ordre des langues utilisées : DEL2, DEL3, DEL4, etc., l'ordre des langues étant celui de leur apparition dans le cursus suivi par les élèves.

\section{Enseignement « bilingue » dans le premier et le second degré en France}

\section{1. Les modèles de DEL2 sur le terrain}

Pour connaître les conceptions d'enseignement d'une discipline ou d'un savoir disciplinaire en langue 2, nous esquisserons les pratiques de classe les plus répandues.

Le concept un maître - une langue a été particulièrement mis en place en France dans toutes les régions bénéficiant de la reconnaissance de leur langue régionale (voir supra), pour les classes dites bilingues à parité horaire des langues depuis la maternelle jusqu'en CM2, parfois appelées classes semi-immersives. Dans les sites bilingues alsaciens, où la moitié des enseignements dans ces classes s'effectue en L1 (français), l'autre moitié en L2 (allemand standard, complété ou non par des cours en dialecte alsacien), on parlera alors d'enseignement bilingue à parité horaire et de langue; il se fait en immersion partielle.

Le concept du maître unique biculturel est prédominant, entre autres, dans l'enseignement primaire au Val d'Aoste ${ }^{6}$. En classe, les langues de travail sont simultanément le français et l'italien; les élèves sont donc scolarisés dans les deux langues ce qui correspond à la politique de maintien et de généralisation du bilinguisme territorial (Cavalli, 2005).

En ce qui concerne le rapport entre la discipline enseignée et la L2, nous rencontrons le modèle intégratif fréquemment au lycée, plus particulièrement dans les classes de la filière bilingue, comme c'est le cas des sections européennes ou de langues orientales au lycée en France. En Suisse par exemple, on parlerait dans ce cas plutôt d'un enseignement disciplinaire bilingue ou d'un programme ou d'un enseignement d'immersion. De nombreuses recherches ont démontré l'efficacité d'un tel enseignement dans les différentes disciplines.

Le modèle centré sur le contenu ${ }^{7}$ est probablement le plus répandu. Nous le rencontrons dans les trois conceptions de la DEL2 qui sont un maitre - une langue; le maître unique biculturel; l'approche plurilingue.

14 Au niveau du curriculum scolaire, ces cours de DEL2 peuvent être dispensés de la manière suivante :

- soit en une filière propre, par exemple celles des classes bilingues (français-allemand) d'un collège ;

- soit sous forme modulaire (c'est-à-dire que la discipline est enseignée en L2 seulement à certaines périodes de la scolarité). Ce modèle est très fréquent dans les établissements 
allemand du secondaire (Gymnasium ou Realschule) ainsi qu'au Pays-Bas, mais quasiment inexistant en France ;

- soit sous une forme mixte, par exemple en Allemagne, l'histoire-géographie prévue en DEL2 dans un lycée pendant deux ans, puis sous forme de modules thématiques, limités à 6 semaines consécutives par exemple, les années suivantes. disciplinaire en langue 1 et 2 :

- La discipline est enseignée exclusivement et de façon continue tout au long de la scolarité en L2 (par exemple l'histoire en section ABIBAC en France de la Seconde à la Terminale) ;

- La discipline est enseignée en L1 et en L2 de façon continue tout au long de la scolarité ; les contenus traités en L1 ne sont pas les mêmes qu'en L2 (par exemple, dans les collèges sites bilingues alsaciens, le programme de mathématiques et celui d'histoire sont répartis sur les deux langues). À l'école primaire, cela est vrai à la marge pour les mathématiques, mais beaucoup plus nettement pour l'EPS, le chant et la musique, les arts plastiques;

- La discipline est enseignée en L1 et en L2 de façon discontinue et modulaire, par exemple dans le cadre de projets, mais pas en France (voir supra).

Les filières en France dans lesquelles des contenus sont dispensés dans une autre langue :

Pour la maternelle et l'élémentaire, des classes dites paritaires accueillent les enfants dont les parents ont exprimé le souhait de les scolariser en « site bilingue ». Ces enfants bénéficient d'un enseignement du programme national français avec un apport dans une langue régionale et des domaines d'activités ou des disciplines enseignées en partie ou intégralement dans cette langue régionale, d'où la dénomination réductrice "dispositif français + langue régionale». Il reste à préciser que ce dispositif n'est possible que pour les langues qui figurent dans la liste citée précédemment, et pour un volume horaire de la langue régionale ne dépassant pas la moitié des cours hebdomadaires $(2 \times 12$ heures hebdomadaires actuellement).

Le numéro 31 de la revue Tréma ${ }^{8}$ a consacré en 2009 un état des lieux de l'enseignement de neuf langues de France et des enjeux actuels. Sont traités dans le numéro le breton, le gallo, le basque, le catalan, l'occitan, le corse, le tahitien et les langues polynésiennes, la langue régionale d'Alsace et des Pays mosellans, le créole réunionnais.

Pour le second degré, nous présenterons les quatre filières les plus empruntées par les élèves en Alsace (Geiger-Jaillet 2005a: 184 ff.). Celles-ci varient considérablement au niveau des objectifs, des prérequis, de l'organisation scolaire et de l'horaire, de l'approche en DEL2 ou pas, des supports utilisés et des résultats.

- Les classes bilingues de collège sont la continuité d'un enseignement dit immersif du premier degré, lié au dispositif des langues régionales. Théoriquement, toutes les disciplines scolaires peuvent être enseignées en allemand, mais concrètement cela dépend des ressources humaines en termes d'enseignants disponibles dans un établissement. Dans certaines régions, les filières bilingues existent en lycée.

- Les sections ABIBAC (allemand), ESABAC (italien) ou BACHIBAC (espagnol) sont une spécialisation à l'intérieur de cette catégorie car elles mènent à un diplôme binational, elles sont proposées en nombre limité de sections et uniquement en lycée.

- Également au niveau du lycée, l'option internationale du baccalauréat OIB qui se prépare uniquement dans des établissements à sections internationales.

- Les Sections européennes ou de langue orientale existent depuis 1992 en collège et lycée ${ }^{9}$; la mention de la section suivie apparaît sur le diplôme du baccalauréat sous certaines 
conditions. Les titulaires de cette mention sont parfois dispensés de certaines formalités d'inscription et d'examens d'entrée de langues dans les universités.

- Afin de permettre aux jeunes l'étude simultanée de deux langues étrangères en 6e, les sections bi-langues (anciennement appelées trilingues) se sont mises en places dans les années 1980. Ces sections proposent un enseignement linguistique renforcé dans deux langues vivantes ( 3 heures hebdomadaires dans chacune des langues en Sixième), mais elles ne prévoient pas d'enseignement en DEL2. Nous n'en tiendrons pas compte ici.

\section{2. Les enseignants de ces filières} l'enseignement des DEL2 en France et en Allemagne " (Geiger-Jaillet, 2005b), sur «Les formations bilingues à l'IUFM d'Alsace : bilan et perspectives » (Geiger-Jaillet \& Morgen, 2007) ou sur « La formation des enseignants en France : histoire, réalités et perspectives actuelles» (Geiger-Jaillet \& Morgen, 2008). Dans un guide présenté par l'Association Lehrer (2008), nous nous sommes exprimés sur ce que devrait être une formation spécifique des enseignants se destinant à l'enseignement bilingue à parité horaire dans le contexte du dispositif alsacien. D'autres chercheurs, eu égard à la composition des classes actuelles, ont complété la liste des publications par des articles abordant les compétences qui semblent spécifiques à un enseignement en contexte plurilingue sans que celui-ci fasse forcément l'objet d'un dispositif spécifique de DEL2 (Young \& Mary, 2009 entre autres).

Pendant de nombreuses années, des enseignants issus de diverses disciplines ont enseigné leur discipline en langue étrangère ou régionale sans avoir bénéficié auparavant d'une formation adaptée (Geiger-Jaillet, 2005a). Cette absence de dispositif n'était d'ailleurs pas propre à la France. La demande de la société (voire des parents) ou d'autres instances (institutionnelles, académiques...) a souvent devancé la mise en place de formations adaptées ou de programmes spécifiques et l'administration ministérielle a ainsi conçu le cadrage en même temps que sa mise en place. Un concours spécifique CRPE spécial de langues régionales pour le professorat des écoles en langue régionale s'est mis en place en 2002, avec les formations (désormais au niveau master) qui y préparent aussi bien pour l'enseignement public que pour le privé. Pour le primaire, la France recrute donc de futurs enseignants en fonction de leurs compétences en mathématiques, en français (langue nationale) et dans la langue régionale de l'académie en question, ainsi que dans d'autres disciplines de l'école primaire.

21 Le lecteur l'aura compris : à côté des langues reconnues dans certaines académies comme «langues régionales» qui peuvent faire l'objet d'un enseignement bilingue dans le premier degré, le choix est élargi aux langues vivantes étrangères pour le second degré. On peut ainsi trouver des professeurs certifiés enseignant les arts plastiques en anglais, ou des agrégés enseignant l'histoire-géographie en allemand. Comment sont-ils formés?

Un parcours possible est celui de choisir un cursus intégré entre deux établissements de l'enseignement supérieur dans deux pays différents. À titre d'exemple, l'université franco-allemande ${ }^{10}$ recense dans une brochure 140 cursus binationaux franco-allemands en 2012-13, mais presque aucun ne concerne l'enseignement ${ }^{11}$.

Pour le secondaire, dans beaucoup de pays - notamment germanophones -, les enseignants sont formés dans deux disciplines en plus des sciences de l'éducation qui constituent la troisième discipline à partir de la première année à l'université. Ces 
formations aboutissent à des diplômes de bivalence, par exemple : français + géographie, allemand + histoire, etc. Certaines combinaisons sont évidemment plus recherchées que d'autres. La France s'est alignée sur les usages de ses voisins européens, en installant des CAPES bivalents pour le basque, le breton, le catalan, le corse et l'occitan, concours dans lesquels une langue régionale présente en France s'ajoute à une autre matière scolaire. Mais en dehors de ces cas, la connaissance spécialisée dans un seul domaine primait jusqu'à présent et les dispositifs des concours de recrutement du second degré n'ont pas évolué vers la bivalence.

Quelques formations universitaires telles que des bi-DEUG allemand / histoire (Paris), des doubles diplômes (Tübingen avec Aix-Marseille) ou des cursus intégrés (Lyon avec Leipzig) existent, mais en amont des concours. Dans le domaine de l'enseignement, les doubles formations se sont mises en place bien après les doubles formations des ingénieurs, des médecins ou des juristes par exemple.

L'encadrement dans les sections européennes est assuré par des enseignants de langues et des enseignants disciplinaires habilités par un inspecteur de langue et nommés sur un poste à profil en section européenne. C'est précisément la pénurie de professeurs pouvant assurer l'enseignement de leur discipline en langue étrangère qui constitue le principal obstacle à la multiplication de ces sections.

À l'IUFM d'Alsace, une formation spécifique (en 2e année) intitulée «formation bilingue complémentaire OEB (option européenne et bilingue) », existait entre 1994 et 2009-2010 pour les professeurs stagiaires des lycées et collèges. Dans les IUFM de Rennes et de Grenoble une telle formation était proposée sous l'appellation « Enseignement d'une discipline non linguistique (DNL) en section européenne (SE) ». Les stagiaires PLC2 post-concours formés en $\mathrm{OEB}^{12}$ à l'IUFM d'Alsace avaient ainsi la possibilité de se qualifier pour enseigner leur discipline en langue étrangère allemand, anglais, espagnol, puis bénéficiaient d'une priorité d'affectation dans l'académie de Strasbourg. Cependant, cette formation complémentaire ne réglait pas le problème de la formation initiale des candidats avant concours ni celui de concours existants non adaptés aux besoins de l'enseignement des DEL2.

Depuis la mise en œuvre des masters aux métiers de l'enseignement, les candidats à l'enseignement $\mathrm{du}$ second degré sont formés uniquement dans leur composante à l'université, avec une toute petite part de didactique dispensée dans la composante IUFM de cette même université. De ce fait, l'ancienne formation bilingue OEB que l'IUFM d'Alsace a mise en place à l'intention des futurs enseignants de DEL2, a disparu depuis 2010 en même temps que celles de Rennes et Grenoble, alors que les besoins en enseignants de mathématiques, d'histoire, de géographie, de sciences en langue allemande, sont énormes dans l'académie de Strasbourg.

Les professeurs du second degré recrutés depuis pour la voie bilingue des collèges et lycées ou les sections européennes n'ont plus aucune formation hors de la formation complémentaire que le Rectorat peut éventuellement leur proposer. Les candidats éventuels se signalent auprès de leur inspecteur, ce qui relève d'une démarche très individuelle.

29 C'est seulement en 2012-2013 que l'IUFM d'Alsace a pu créer un diplôme d'université (DU) «Enseigner sa discipline en allemand», mais celui-ci est pour l'instant réservé aux enseignants déjà en poste en Alsace, dans le cadre de la formation continue (voir infra). 
Nous constatons un manque de cadrage national au niveau de concours bivalents et de formations DEL2. De plus, il y a absence d'un certain corporatisme des enseignants pour se sentir "professeur de DEL2 " et donc de revendiquer une appartenance à une discipline ET une langue. Il n'est pas étonnant que l'on n'ait pas créé en France des associations d'enseignants spécifiques de ce type, contrairement à l'Allemagne.

Des associations d'enseignants bilingues existent un peu partout en France, à l'instar de l'association LEHRER (qui regroupe des enseignants de classe bilingue français-allemand en Alsace et en Moselle) ou de la FLAREP. Comme son nom l'indique l'ADEB (Association pour le Développement de l'Enseignement Bi/Plurilingue) est une association de chercheurs, mais elle ne s'occupe pas que de l'enseignement.

\section{2. 1. Publications ${ }^{13}$ sur l'enseignement des DEL2}

Pour le second degré, la diversité des filières, des niveaux (collège et lycée), des disciplines (pratiquement toutes les disciplines enseignées), et le nombre relativement peu élevé d'élèves qui fréquentent ces dispositifs en France pour les raisons évoquées expliquent le fait que peu de publications françaises tentent de présenter une vue d'ensemble de ce qu'est le statut et de ce que sont les fonctions et les pratiques pédagogiques des professeurs des DEL2. La revue Langues Modernes a consacré un numéro en 2009 à « Enseigner des disciplines non linguistiques (DNL) »; une publication de 2011 (Duverger, 2011) rédigée par l'Association pour le Développement de l'Enseignement Bi/Plurilingue avec la contribution de plusieurs auteurs est disponible gratuitement en ligne. Rappelons également notre ouvrage portant sur la didactique et la méthodologie des DEL2 : Enseigner une discipline dans une autre langue : méthodologie et pratiques professionnelles (Geiger-Jaillet, Schlemminger \& Le Pape Racine, 2011). Mentionnons efin les ouvrages suisses de langue française qui peuvent être une source d'information utiles : C. Brohy (dir.) (2012) : Zweisprachiger Unterricht: Module, Ausbildung, Nachhaltigkeit. L'enseignement bilingue: Modèles, formation, continuité, ainsi que G. Steffen (2013): Les disciplines dans l'enseignement bilingue. Apprentissage intégré des savoirs disciplinaires et linguistiques ${ }^{14}$.

3 La bivalence des diplômes d'enseignement en Allemagne et la plus grande importance accordée à la didactique expliquent l'existence de publications - souvent des thèses ayant comme objet un croisement de disciplines et de langues. Pour les disciplines enseignées en anglais en Allemagne, on peut citer parmi les ouvrages les plus récents :

- L'histoire : E. Müller-Schneck (2006);

- La géographie : C. Schönfeldt (2005);

- L'éducation physique et sportive : B. Rottmann (2006);

- Les sciences sociales : S. Wolf (2006);

- La chimie : A. Bonnet (2004).

Pour les disciplines enseignées en français, toujours chez les élèves germanophones, nous pouvons citer :

- L'histoire : O. Stern et al. (1999), B. Helbig (2001), S. Lamsfuss-Schenk (2008) ;

- L'économie sociale et familiale : S. Rapp (2006) ;

- Les arts plastiques : S. Witzigmann (2011).

D'autres ouvrages tels que W. Zydatiss (2007), S. Doff (2010), B. Diehr \& L. Schmelter (2012) traitent des DEL2 de façon plus générale dans l'enseignement secondaire allemand ou en Suisse alémanique (Badertscher, 2005). Des congrès sont d'ailleurs très 
régulièrement consacrés à l'enseignement et l'apprentissage en contexte bi- ou plurilingue.

\section{2. 2. Présentation du nouveau dispositif de formation continue en Alsace} allemande » en 2013 à l'université de Strasbourg doit permettre d'alimenter le vivier d'enseignants formés pour assumer cette double mission : enseigner une discipline dans une langue autre que le français dans les classes ou sections bilingues et les sections européennes ou internationales. Ce vivier s'est tari depuis la mise en place de la formation en master des enseignants, car il n'a pas été possible d'implanter au sein des différentes composantes de l'université de Strasbourg un parcours spécifique répondant aux besoins de l'académie. C'est donc une réponse de l'université de Strasbourg via l'IUFM puis des Écoles supérieures du professorat et de l'éducation ESPE, depuis septembre 2013, afin de contribuer à la politique linguistique de la Région Alsace et de 1 'Académie de Strasbourg.

s'agit d'un DU pour des enseignants déjà en poste qui voudraient se former à enseigner leur discipline en allemand. La mise en place d'un même DU pour l'anglais est en discussion. Les enseignants de langue, sans autre formation disciplinaire, sont exclus de cette formation. La première promotion de février à octobre 2013 comprend une quinzaine d'enseignants représentant huit disciplines différentes en collège ou lycée. Après l'obtention de leur DU, les enseignants formés bénéficieront d'autres actions de formation continue dans le cadre du PAF (Plan Académique de Formation ou plan départemental de formation).

\section{2. 3. Les caractéristiques d'un DU}

Les DU sont des diplômes portés par une université qui doivent entièrement s'autofinancer. Parmi les constantes de financement qu'a déjà révélé le numéro $n^{\circ} 31$ de la revue TRÉMA (IUFM Montpellier) dans le contexte de langues régionales en France, c'est bien la collectivité territoriale qu'il faut mentionner en premier lieu. Celle-ci peut être soit une région, soit un département, soit un regroupement de communes, soit une émanation de ces instances. La création d'un Groupement d'intérêt public, l'office public de la langue basque (voir : OPLB, en ligne : http://www.mintzaira.fr/), en est l'exemple le plus significatif ${ }^{15}$. Ailleurs, c'est la Région (Alsace, Aquitaine, Bretagne) ou la Collectivité territoriale de Corse, dotée de prérogatives supplémentaires, qui assure ce suivi en partenariat avec le Recteur.

té de l'université de Strasbourg et de l'IUFM, le DU alsacien associe l'Institut Goethe de Paris avec sa compétence en DEL2 ainsi que deux établissements supérieurs allemands de formation des enseignants qui sont distants d'une heure de route chacun de Strasbourg (les deux Pädagogische Hochschulen à Freiburg et Karlsruhe). L'équipe enseignante est issue de l'ESPE et des institutions partenaires.

Le DU vise des enseignants en poste qui souhaitent poser leur candidature à un poste à profil (sections bilingues, sections européennes, OIB, classes ABIBAC). En termes de prérequis, il faut satisfaire à un test de langue allemande d'un niveau $\mathrm{B} 2-\mathrm{C} 1$ du cadre européen commun de référence pour les langues. 
- 50h de double didactique (disciplinaire + linguistique) ;

- $20 \mathrm{~h}$ de perfectionnement linguistique ;

- 20 h d'autoformation ;

- $20 \mathrm{~h}$ de stage (observation d'heures de cours dans l'Académie et dans les régions de Freiburg et/ou Karlsruhe et une participation à un module de formation à Freiburg).

Comme la formation s'adresse à des enseignants en poste, le temps de formation se concentre sur des vendredis après-midis et des samedis matins (les emplois du temps doivent être faits en conséquence) ainsi qu'une semaine des vacances scolaires en février et la première semaine de juillet.

Les objectifs pédagogiques et professionnels sont fixés dans la maquette lors de création du DU (document interne Université de Strasbourg, 2013) et se trouvent sur le site de l'université17 :

\section{«Objectifs pédagogiques et scientifiques}

- Consolidation du niveau de la langue cible ;

- Connaissance des principales formes et conditions d'un enseignement d'une discipline en langue 2 ;

- Connaissance de quelques aspects de l'appropriation d'une L2 ;

- Gestion des interactions en classe de DEL2 et construction intégrée des savoirs et savoirfaire disciplinaires et linguistiques.

\section{Objectifs en terme de compétences professionnelles}

- Savoir formuler pour une DEL2 donnée ses attentes et ses objectifs sur le plan linguistique et disciplinaire ;

- Savoir transposer des contenus disciplinaires dans une langue étrangère ;

- Savoir distinguer procédés et techniques propres à soutenir des apprentissages disciplinaires et propres à l'enseignement des langues ;

- Savoir recourir à des approches issues de la didactique des langues pour favoriser l'apprentissage en action de la langue ;

- Savoir prendre en compte les défis linguistiques dans la construction de la progression disciplinaire ;

- Connaître et savoir analyser des bonnes pratiques ;

- Connaître le statut de l'erreur dans l'acquisition d'une langue et plus particulièrement en DEL2, savoir analyser le type d'erreurs identifiées et savoir corriger de façon appropriée les erreurs à l'oral comme à l'écrit ;

- Savoir prendre en compte la double acquisition dans la conception du dispositif d'évaluation;

- Savoir travailler en réseau dans une perspective d'apprentissage tout au long de la vie ».

Perspectives d'évolution : la réforme de la formation des enseignants au sein des écoles supérieures du professorat et de l'éducation (ESPE).

Pour la rentrée 2013-14, de nouvelles maquettes de master ont vu le jour. Cette fois-ci, c'est la nouvelle ESPE de l'académie de Strasbourg qui a la responsabilité de toutes les 
maquettes de master liées à la formation des futurs enseignants, qu'il s'agisse des formations du premier ou du second degré.

Les impératifs ministériels de réduction des heures de maquette ont été mis en application et la part des langues vivantes dans le tronc commun fortement réduite et de surcroît limitée la seule année de M2. Puisqu'il n'est pas question pour l'instant d'ouvrir un nouveau master pour l'enseignement des DEL2, la seule possibilité de proposer un début de formation initiale serait d'identifier ceux parmi les futurs enseignants qui se sentiraient capables d'enseigner leur discipline dans une autre langue que le français. $\mathrm{Au}$ lieu de suivre les heures obligatoires en LV, ils pourraient bénéficier du même volume horaire pour une initiation à l'enseignement des DEL. Ce montage se situerait en formation initiale (niveau master 2), les frais seraient couverts par les frais d'inscription des étudiants ${ }^{18}$ et le budget de l'université de Strasbourg, ce qui permettrait une ouverture vers d'autres langues que l'allemand, par exemple l'anglais et l'espagnol. Car l'un des soucis actuels est le financement de ce DU: les collectivités territoriales soutiennent certes ces enseignements disciplinaires en allemand, mais elles sont plus difficiles à convaincre quand il s'agit de DEL anglais ou espagnol.

De plus, le dispositif actuel de DU en formation continue n'a qu'un impact limité face à la pénurie d'enseignants de DEL du fait qu'elle ne peut s'adresser uniquement à des enseignants déjà en poste dans l'académie. Ce DU ne permet malheureusement pas d'attirer d'éventuels candidats en Alsace pour suivre une telle formation, ou de susciter des vocations en amont.

51 Enfin, les effectifs restent faibles, car dans le meilleur des cas, deux ou trois personnes par promotion enseignent la même discipline, mais en quelle langue dispenser une formation pour un enseignant de mathématiques en langue anglaise et son collègue disciplinaire enseignant en allemand ? L'expérience de l'ancienne formation OEB montre que la langue commune n'était malheureusement que... le français. Or, une formation en langue cible pour un enseignement de DEL2 est plus efficace si on arrive à mettre les enseignants euxmêmes dans un bain linguistique pendant leur formation, ce qui inclut de les familiariser avec les spécificités disciplinaires propres à la culture de la langue cible.

Rêvons un peu : si Strasbourg, Rennes et Grenoble proposaient autrefois des formations initiales pour quasiment toute la France, il serait peut-être possible de créer à présent des spécialisations par "pôle ", par exemple l'espagnol à Bordeaux, le catalan à Perpignan, l'anglais dans la région parisienne et l'allemand en Alsace? Une telle concentration par langue permettrait de créer des liens entre les enseignants de DEL2 au-delà des disciplines enseignées et de rentabiliser la formation.

Des sessions de formations continues ou des décharges seront de toute façon nécessaires pour produire le matériel de base pour un bon enseignement de DEL2 et couvrir largement les disciplines. Certains sites y sont d'ailleurs déjà dédiés. Suite à l'expérience du DU alsacien, il est par ailleurs envisagé de constituer un réseau d'enseignants titulaires de ce diplôme d'université qui se rencontreraient deux fois par an pour des échanges de bonnes pratiques et de supports et pour élaborer des documents utiles à 1 'ensemble des enseignants de DEL2. 


\section{Conclusion}

Nous avons présenté dans ce texte le nouveau diplôme d'université consacré à la formation «Enseigner sa discipline en langue allemande ». Trois points nous paraissent fondamentaux.

- Puisque les formations à l'enseignement bilingue dans le premier degré sont en général assurées dans les académies avec une langue régionale reconnue, il convient dorénavant de faire repartir une formation des professeurs de collège et de lycée. Le moment est à saisir par les nouvelles ESPE.

- Pour installer de nouvelles formations pour l'enseignement des disciplines enseignées dans une autre langue que la langue de la scolarité, nous proposons de prendre appui sur la maquette du DU existant. Mais une réflexion s'impose pour adapter à la formation initiale bilingue un dispositif conçu pour la formation continue. Cette réflexion pourrait, dans notre esprit, associer les ESPE concernées par cette problématique.

- Dans ce cadre, nous proposons des pôles de formation centrés sur la langue de formation que chaque partenaire connaît le mieux, l'allemand, l'anglais, l'espagnol...

- Pour éviter une nouvelle fois de laisser les langues régionales à la marge de cette réflexion, on pourrait moduler ces formations autour de pôles d'enseignement de langues germaniques, pôle d'enseignement de langues romanes.

- Enfin, le moment serait opportun pour mettre en commun la réflexion sur toutes ces avancées, au sein d'un groupe de recherche formation commun aux ESPE en France.

\section{BIBLIOGRAPHIE}

ADEB (2011). Enseignement bilingue : Le professeur de « Discipline Non Linguistique » (DNL). Brochure rédigée et éditée par l'ADEB en décembre 2011, disponible en ligne gratuitement http://www.adeb.asso.fr/publications_adeb/ADEB_brochure_DNL_12_2011.pdf (dernière consultation : 30/08/2013).

Association LEHRER (éd.) (2008). Ich möchte Lehrer werden ... devenir enseignant bilingue. Guide de l'enseignant(e) en classe bilingue paritaire français - langue régionale de l'académie de Strasbourg. (Info-Lehrer hors série). ISSN 1283-8314

Badertscher, H. (2005). Wissenserwerb im bilingualen Unterricht. Eine empirische Studie zur Bedeutung der Sprache im Sachlernen, CLIL. Schlussbericht, Bern : Universität Bern. Institut für Pädagogik und Schulpädagogik

Bodenbender, V. \& Dietrich-Chenel, K. (2013). « 15 ans de formation franco-allemande de professeurs dans la région du Rhin supérieur : la traversée des frontières et ses limites ». Synergies pays germanophones $n^{\circ} 6,83-93$.

Bothorel-Witz A., Morgen D. \& Colinet J.-C. (2004). Enseigner en classe bilingue. Former les enseignants des classes bilingues français-langues régionales. Actes de l'Université d'automne du 
24 au 27 octobre 2002 à Guebwiller (IUFM d'Alsace). Ministère de l'Éducation nationale. Direction de l'enseignement scolaire. Juin 2004. (pages 261 à 275).

Brohy, C. (dir.) (2012). Zweisprachiger Unterricht: Module, Ausbildung, Nachhaltigkeit. L'enseignement bilingue : Modèles, formation, continuité. Fribourg. Université de Fribourg.

En ligne sur le site de l'APEPS : http://www.plurilingua.ch/userfiles/file/AktenZUGAPEPS-2010-12-12.pdf (dernière consultation: 30/08/2013).

Diplôme d'Université (DU) Enseigner sa discipline en langue allemande / Université de Strasbourg. En ligne sur le site de l'université : http://espe.unistra.fr/etudes-et-scolarite/m2annee-transitoire/diplome-duniversite-du-enseigner-sa-discipline-en-langue-allemande/ (dernière consultation: 30/08/2013).

Doff, S. (2010). Bilingualer Sachfachunterricht in der Sekundarstufe: Eine Einführung, Gunter Narr.

Duverger, J. (dir.) (2011). Enseignement bilingue. Le professeur de « Discipline Non Linguistique ». Statut, fonctions, pratiques pédagogiques. Paris : Association pour le Développement de l'Enseignement Bi/Plurilingue. En ligne sur le site de l'association : http:// www.adeb.asso.fr/publications_adeb/ADEB_brochure_DNL_12_2011.pdf (dernière consultation : 30/08/2013).

Geiger-Jaillet A., Schlemminger, G. \& Le Pape Racine, C. (2011). Enseigner une discipline dans une autre langue : méthodologie et pratiques professionnelles, Peter Lang.

Geiger-Jaillet A. \& Morgen, D. (2008). La formation des enseignants en France : histoire, réalités et perspectives actuelles, 333-357. In Französisch heute $\mathrm{N}^{\circ} 4 / 2008$.

Geiger-Jaillet A. \& Morgen D. (2007). Bilinguale Ausbildungsgänge für das Lehramt im Elsass: Bilanz und Perspektiven, 41-61. In Geiger-Jaillet, A. ; Lehren und Lernen in einer Grenzregion: Schwerpunkt Oberrhein, Baltmannsweiler : Schneider-Verlag Hohengehren.

Geiger-Jaillet A. (2005a). Le bilinguisme pour grandir. Naître bilingue ou le devenir par l'école. Paris : L'Harmattan.

Geiger-Jaillet A. (2005b). Mehrsprachige Lehrerbildung für bilingualen Sachfachunterricht in Deutschland und Frankreich, 81-97. In Denk, R. (éd.), Nach Europa unterwegs:

Grenzüberschreitende Modelle der Lehrerbildung im Zeichen von europäischer Identität, Kultur und Mehrsprachigkeit. Pfaffenweiler : Centaurus-Verlag.

Hollm, J., Hüttemann, a., Kessler, J.-U., Schlemminger g. \& Ade-Thurow, B. (dir.) (2013).

Bilinguales Lehren und Lernen in der Sekundarstufe 1: Sprache, Sachfach und Schulorganisation. Landau : Verl. Empirische Pädagogik.

Les Langues Modernes « Enseigner des disciplines non linguistiques (DNL)», n³, 2009.

L'enseignement des langues régionales en France aujourd'hui : état des lieux et perspectives. Revue Tréma (IUFM Montpellier) N³1/ septembre 2009.

MEN (Ministère d'Education nationale). Modalités d'organisation du concours externe et du second concours interne de recrutement de professeurs des écoles. Arrêté du 3-1-2002 ;

JO du 5-1-2002. En ligne sur le site du ministère :

http://www.education.gouv.fr/botexte/bo020214/MENP0102487A.htm (dernière consultation : 30/08/2013).

Schlemminger, G. (2008). « Une approche didactique de l'enseignement bilingue : le modèle rhénan », Synergies Pays Germanophones 2008, 97-111. 
Steffen, G. (2013). Les disciplines dans l'enseignement bilingue. Apprentissage intégré des savoirs disciplinaires et linguistiques. Frankf. et al : Peter Lang.

Université franco-allemande (éd.) Cursus intégrés franco-allemands 2012-13. Brochure. http:// www.dfh-ufa.org/uploads/media/DFH_Studienfolder_F_05_13_web_02.pdf (dernière consultation: 30/08/2013).

Young, A. \& Mary, L. (2009). Former les professeurs stagiaires à la diversité linguistique à l'école, Le français aujourd'hui 1/2009 ( $\left.\mathrm{n}^{\circ} 164\right)$, p. 87-97. En ligne : www.cairn.info/revue-le-francaisaujourd-hui-2009-1-page-87.htm. (dernière consultation : 30/08/2013).

\section{Références des sites Internet}

Association LEHRER (Association des enseignants des classes bilingues français-langue régionale dans les académies de Strasbourg et Nancy-Metz).

En ligne : http://associationlehrer.free.fr/ (dernière consultation : 30/08/2013).

Association pour le Développement de l'Enseignement Bi/Plurilingue (ADEB).

En ligne : http://www.adeb.asso.fr/ (dernière consultation : 30/08/2013).

ESPE de l'académie de Strasbourg.

En ligne : http://espe.unistra.fr/linstitution/espe-ecole-superieure-du-professorat-et-deleducation/ (dernière consultation : 30/08/2013).

EURODNL (site d'échange collaboratif, destiné aux professeurs de l'enseignement bilingue, documents pour l'allemand, l'anglais, l'espagnol, l'italien.

En ligne: http://www.ac-nancy-metz.fr/stage/archambault/eurodnl/default.htm (dernière consultation : $30 / 08 / 2013)$.

Fédération pour les Langues Régionales dans l'enseignement public (FLAREP).

En ligne : http://www.flarep.com/ (dernière consultation : 30/08/2013).

Institut Supérieur des Langues de la République Française (ISLRF) : établissement d'enseignement supérieur associatif qui fédère cinq réseaux d'écoles en langue dites régionales : En ligne : http:// aprene.org/node/187 (dernière consultation : 30/08/2013).

\section{NOTES}

1. Le Lycée franco-allemand de Sarrebruck en Sarre (RFA), ancien Lycée français Maréchal Ney, est créé en 1961, avant le Traité de l'Elysée.

2. http://www.education.gouv.fr/botexte/bo020214/MENP0102487A.htm

3. Il y a les Sections européennes et orientales suivantes : allemand, anglais, espagnol, italien, néerlandais, portugais, russe. Sections de langues orientales : arabe, chinois, japonais, vietnamien (Voir: "Sections européennes ou de langues orientales" sur le portail du Ministère de l'éducation nationale : http://eduscol.education.fr/cid46516/indication-section-europeenne-ousection-de-langue-orientale-au-baccalaureat.html (dernière consultation : 28/08/2013).

4. On dénombre actuellement 28 Gymnasien en Allemagne et autant de lycées en France.

5. Il n'y a pas lieu de présenter et de discuter les différents signes et abréviations concernant ce domaine, comme CLIL, EMILE, DdNL, etc. ; voir à ce sujet A. Geiger-Jaillet, G. Schlemminger \& C. Le Pape Racine (2011: 20 et s.). 
6. Dans le secondaire, le concept « un maître - une langue » est pratiqué en suivant le modèle intégré de la DEL2.

7. Pour sa conceptualisation, voir aussi G. Schlemminger (2008).

8. L'enseignement des langues régionales en France aujourd'hui : état des lieux et perspectives. Revue Tréma (IUFM Montpellier) $\mathrm{N}^{\circ} 31$ / septembre 2009. En ligne: http:// www.montpellier.iufm.fr/internet/site/ (dernière consultation : 28/08/2013).

9. La mention est définie par arrêté du 9 juin 2003 (BO n² 24 du 12 juin 2003).

10. http://www.dfh-ufa.org/

11. Pour le premier degré, la seule exception est le cursus intégré entre Freiburg (D) et Mulhouse/Strasbourg (F), voir l'article dans «Synergies des pays germanophones » de V. Bodenbender \& K. Dietrich-Chenel (2013) ou le site Internet https://www.ph-freiburg.de/ projekte/its/startseite.html (dernière consultation : 28/08/2013).

12. Entre 1994-95 et 2009-10, 241 lauréats du CAPES ou de l'agrégation ont obtenu la certification OEB pour sept disciplines dans une des trois langues (allemand, anglais, espagnol) à l'IUFM d'Alsace, dont 165 pour l'allemand.

13. Nous ne présentons ici que les publications en langue française et allemande. Les publications en langue anglaise, étant très nombreuses, mériteraient une présentation à part.

14. Pour l'Allemagne, la publication concernant la mise en place des filières bilingues dans les collèges du Bade-Wurtemberg mérite d'être mentionnée : J. Hollm, A. Hüttemann,, J.-U. Kessler, G. Schlemminger \& B.-J. Ade-Thurow (dir.) (2013).

15. L'OPLB - qui associe étroitement l'État et les collectivités - gère le développement de la langue basque et la carte scolaire des enseignements.

16. ESPE de l'académie de Strasbourg, voir: http://espe.unistra.fr/linstitution/espe-ecolesuperieure-du-professorat-et-de-leducation/ (dernière consultation : 30/08/2013).

17. Voir : http://espe.unistra.fr/etudes-et-scolarite/m2-annee-transitoire/diplome-duniversitedu-enseigner-sa-discipline-en-langue-allemande/\#c5803 (dernière consultation : 30/08/2013).

18. Les frais d'inscription à l'université se chiffrent autour de 200 ou $300 €$, contrairement aux $1800 €$ de la formation en DU.

\section{RÉSUMÉS}

À partir de l'enseignement bilingue existant en France (modèles, enseignants, publications en didactique), nous ferons un petit historique de l'enseignement et la formation bilingue du second degré en Alsace. Ensuite, nous présenterons le nouveau dispositif de formation continue « Enseigner sa discipline en allemand », ses contenus et les choix faits en Alsace en 2013.

From the existing bilingual education in France (models, teachers, educational publications), we will make a little history of education and bilingual training in secondary school in Alsace. Then we present the new device training "Teaching German discipline" its content and the choices made in Alsace in 2013. 
INDEX

Mots-clés : diplôme d'université, discipline enseignée en langue 2, dispositif de formation, filière bilingue

Keywords : bilingual instruction, discipline taught in a foreign language, teacher training plans, university diploma

\section{AUTEURS}

ANEMONE GEIGER-JAILLET

Professeur des Universités (sciences du langage), Université de Strasbourg, Enseignement bilingue et allemand précoce, EA 1339 LILPA, équipe GEPE,(groupe d'études sur le plurilinguisme en Europe)

\section{GÉRALD SCHLEMMINGER}

Professeur des Universités (sciences du langage), École supérieure de pédagogie de Karlsruhe, Enseignement du FLE et bilingue, Centre du bilinguisme scolaire 